\title{
Flow pack facets of the single node fixed-charge flow polytope
}

\author{
Alper Atamtürk ${ }^{1}$ \\ Department of Industrial Engineering and Operations Research, University of California at Berkeley, Berkeley, \\ CA 94720-1777, USA
}

Received 1 December 2000; received in revised form 1 June 2001; accepted 5 July 2001

\begin{abstract}
We present a class of facet-defining inequalities for the single node fixed-charge flow polytope and provide a comparison of valid inequalities for this polytope. We also present computational results that show the effectiveness of these inequalities in solving fixed-charge network flow problems. (c) 2001 Elsevier Science B.V. All rights reserved.
\end{abstract}

\section{Introduction}

The single node fixed-charge flow model is a basic structure that arises as an important relaxation of many mixed 0-1 integer programming (MBIP) problems with fixed charges. The model consists of a flow balance inequality for a single node with demand $b$ and variable upper bounds on inflow and outflow arcs, which can be formulated as

$$
S=\left\{x \in\{0,1\}^{|N|}, y \in \mathbb{R}_{+}^{|N|}: \sum_{i \in N^{+}} y_{i}-\sum_{i \in N^{-}} y_{i} \leqslant b, y_{i} \leqslant u_{i} x_{i} \quad \forall i \in N\right\},
$$

where variable $y_{i}$ is the flow on arc $i$ with capacity $u_{i}, x_{i}$ is a binary variable that indicates whether arc $i$ is open or closed, and $N=N^{+} \cup N^{-}$.

The single node fixed-charge flow model is interesting not only because it is a relaxation of the fixed-charge network flow problem, but also because it is possible to derive relaxations of the form $S$ of a general MBIP problem. Therefore, valid inequalities for $S$ can be used as cutting planes in branch-and-cut algorithms to solve MBIP problems. General purpose mixed-integer programming software packages, such as CPLEX, ${ }^{2}$ MINTO [8] and bc-opt [4] use cutting planes derived from $S$ among others. We refer the reader to [13] for a detailed discussion on using $S$ and related structures as relaxations in mixed-integer programming. Valid inequalities for $S$ have also been instrumental in developing strong cutting planes for a variety of problems, including lot-sizing problems [3,10] and facility location problems [1].

\footnotetext{
${ }^{1}$ Supported, in part, by NSF grants DMI-9908705 and DMI-0070127.

E-mail address: atamturk@ieor.berkeley.edu (A. Atamtürk).

${ }^{2}$ CPLEX is a trademark of ILOG, Inc.
} 
The study of the polyhedral structure of the convex hull of $S$ is initiated by Padberg et al. [9] for the case with $N^{-}=\emptyset$. They introduce the flow cover inequalities, which are generalized by Van Roy and Wolsey [12]. $\mathrm{Gu}$ et al. [5] strengthen these inequalities through sequence independent lifting for $S$. Stallaert [11] gives a complementary class of flow cover inequalities. Marchand and Wolsey [7] derive other inequalities for $S$ from mixed $0-1$ knapsack relaxations of $S$.

Here we introduce yet another class of strong inequalities for $S$ and show the relationship between these inequalities and the ones introduced earlier. In Section 2, we present the flow pack inequalities for $S$ and study their strength for a certain restriction of $S$. Then we strengthen and generalize them by lifting and compare them with other inequalities for $S$ given in the literature. In Section 3, we conclude with a summary of computational experiments that show the effectiveness of the new inequalities in solving capacitated fixed-charge network flow problems with a branch-and-cut algorithm.

\section{Flow pack facets}

Without loss of generality, we assume that (A.1) $u_{i}>0$ for all $i \in N$ (otherwise $y_{i}=0$ ), (A.2) $b+$ $\sum_{i \in N^{-}} u_{i} \geqslant u_{k}$ for all $k \in N^{-}$(otherwise $x_{k}=1$ ), and (A.3) $b+\sum_{i \in N^{-}} u_{i}>0$ (otherwise $y_{i}=0$ for all $i \in N^{+}$). Note that, if $N^{-} \neq \emptyset$, then (A.3) follows from (A.1) and (A.2). Under these assumptions, $\operatorname{conv}(S)$ is full-dimensional. The linear relaxation of $S$ is

$$
P=\left\{(x, y) \in \mathbb{R}_{+}^{2|N|}: \sum_{i \in N^{+}} y_{i}-\sum_{i \in N^{-}} y_{i} \leqslant b, y_{i} \leqslant u_{i} x_{i}, x_{i} \leqslant 1 \quad \forall i \in N\right\} .
$$

Observe that an extreme point of $P$ has at the most one fractional variable in $x$. For $C^{+} \subseteq N^{+}$and $C^{-} \subseteq N^{-},\left(C^{+}, C^{-}\right)$is called a flow cover if $\sum_{i \in C^{+}} u_{i}-\sum_{i \in C^{-}} u_{i}=b+\lambda$ with $\lambda>0$. A fractional extreme point $(\bar{x}, \bar{y})$ of $P$ has $\bar{y}_{k}=u_{k}-\lambda, \bar{x}_{k}=\left(u_{k}-\lambda\right) / u_{k}$ for $k \in C^{+}$, or $\bar{y}_{k}=\lambda, \bar{x}_{k}=\lambda / u_{k}$ for $k \in L^{-} \subseteq N^{-} \backslash C^{-}$with $\lambda<u_{k}$. The rest of the flow and variable upper bound variables in the cover are at their upper bounds, the flow variables not in the cover are at their lower bounds and the remaining variable upper bound variables are at either of their bounds. Letting $K=N^{-} \backslash\left(C^{-} \cup L^{-}\right)$, the well-known flow cover inequality

$$
\sum_{i \in C^{+}}\left(y_{i}+\left(u_{i}-\lambda\right)^{+}\left(1-x_{i}\right)\right)-\sum_{i \in L^{-}} \min \left\{u_{i}, \lambda\right\} x_{i}-\sum_{i \in K} y_{i} \leqslant b+\sum_{i \in C^{-}} u_{i},
$$

where $a^{+}=\max \{a, 0\}$, cuts off the fractional extreme points characterized by flow cover $\left(C^{+}, C^{-}\right)$. If $x_{k}, k \in C^{+}$ is fractional, then (1) is violated by $\left(u_{k}-\lambda\right) \lambda / u_{k}$, else if $x_{k}, k \in L^{-}$is fractional, then it is violated by $\lambda\left(1-\lambda / u_{k}\right)$. Van Roy and Wolsey [12] show that flow cover inequality (1) is facet-defining for $\operatorname{conv}(S)$ if $\max _{i \in C^{+}} u_{i}>\lambda, u_{i}>\lambda$ for all $i \in L^{-}$and $C^{-}=\emptyset$.

An alternative characterization of the fractional extreme points of $P$ gives rise to a different class of valid inequalities for $S$. For $C^{+} \subseteq N^{+}$and $C^{-} \subseteq N^{-},\left(C^{+}, C^{-}\right)$is called a flow pack if $\sum_{i \in C^{+}} u_{i}-$ $\sum_{i \in C^{-}} u_{i}+\mu=b$ with $\mu>0$. A fractional extreme point of $P$ has $\bar{y}_{k}=\mu, \bar{x}_{k}=\mu / u_{k}$ for $k \in L^{+} \subseteq N^{+} \backslash C^{+}$, or $\bar{y}_{k}=u_{k}-\mu, \bar{x}_{k}=\left(u_{k}-\mu\right) / u_{k}$ for $k \in C^{-}$with $\mu<u_{k}$. The rest of the flow and variable upper bound variables in the pack are at their upper bounds, the flow variables not in the pack are at their lower bounds and the remaining variable upper bound variables are at either of their bounds. Letting $K=N^{-} \backslash C^{-}$, the flow pack inequality

$$
\sum_{i \in C^{+}} y_{i}+\sum_{i \in L^{+}}\left(y_{i}-\min \left\{u_{i}, \mu\right\} x_{i}\right)+\sum_{i \in C^{-}}\left(u_{i}-\mu\right)^{+}\left(1-x_{i}\right)-\sum_{i \in K} y_{i} \leqslant \sum_{i \in C^{+}} u_{i}
$$

cuts off the fractional extreme points characterized by flow pack $\left(C^{+}, C^{-}\right)$. If $x_{k}, k \in L^{+}$is fractional, then (2) is violated by $\mu\left(1-\mu / u_{k}\right)$, else if $x_{k}, k \in C^{-}$is fractional, then it is violated by $\left(u_{k}-\mu\right) \mu / u_{k}$. The flow pack inequality is a special case of the inequalities given in Stallaert [11] and may be viewed as a flow cover 
inequality (1) for the relaxation of $S$, where the balance constraint is relaxed to $\sum_{i \in N^{-}} y_{i}-\sum_{i \in N^{+}} y_{i}-s \leqslant-b$ after introducing a slack variable $s$. Flow pack inequalities are facet-defining for the convex hull of the restriction $S_{C^{+}}=\left\{(x, y) \in S: x_{i}=1\right.$ for $\left.i \in C^{+}\right\}$of $S$.

Proposition 1. The following are necessary and sufficient conditions for flow pack inequality (2) to be facet-defining for $\operatorname{conv}\left(S_{C^{+}}\right)$:

1. If $\left|C^{+} \cup K\right| \geqslant 2$, then (a) $u_{i}>\mu$ for all $i \in L^{+}$, and (b) either $L^{+} \neq \emptyset$ or $b+\sum_{i \in N^{-}} u_{i}>u_{k}>\mu$ for some $k \in C^{-}$.

2. If $\left|C^{+} \cup K\right|=1$, then (a) $u_{i}>\mu$ for all $i \in L^{+}$, and (b) either $L^{+} \neq \emptyset$ or $u_{k}>\mu$ for some $k \in C^{-}$.

3. If $\left|C^{+} \cup K\right|=0$, then $\left|L^{+}\right|=1$ and $u_{i} \geqslant \mu$ for $i \in L^{+}$.

Proof. We show only necessity here. For sufficiency, see [2]. Suppose $\left|C^{+} \cup K\right| \geqslant 1$. Let $\zeta=\sum_{i \in C^{+} \cup K} u_{i}$ and $\delta_{i}^{j}=\left(u_{j}-\mu\right) u_{i} / \zeta$. If $u_{i} \leqslant \mu$ for some $i \in L^{+}$, then the inequality obtained by adding $y_{i} \leqslant u_{i} x_{i}$ and the flow pack inequality with $L^{+} \backslash\{i\}$ is at least as strong as the original inequality. Now suppose $L^{+}=\emptyset$. If there is no $k \in C^{-}$with $u_{k}>\mu$, then the flow pack inequality can be obtained by adding $y_{i} \leqslant u_{i}, i \in C^{+}$and $-y_{i} \leqslant 0, i \in K$. Finally, suppose no $k \in C^{-}$with $u_{k}>\mu$ satisfies $b+\sum_{i \in N^{-}} u_{i}>u_{k}$. Since by assumption (A.2), $b+\sum_{i \in N^{-}} u_{i} \geqslant u_{k}$ holds for all $k \in N^{-}, b+\sum_{i \in N^{-}} u_{i}=u_{k}$ for all $k \in C^{-}$with $u_{k}>\mu$. Then $b+$ $\sum_{i \in N^{-}} u_{i}=u_{k} \Leftrightarrow \zeta=u_{k}-\mu \Leftrightarrow u_{i}=\delta_{i}^{k}$ for all $i \in C^{+} \cup K$. But whenever $x_{k}=0$ for $k \in C^{-}$with $u_{k}>\mu, y_{i}=0$ for all $i \in C^{+}$and $y_{i}=u_{i}$ for all $i \in K$ in any feasible solution. Hence if $\left|C^{+} \cup K\right| \geqslant 2$, then there are not enough points on the face defined by (2). If $\left|C^{+} \cup K\right|=0$, then (2) reduces to a trivial inequality $y_{i}-\mu x_{i} \leqslant 0, i \in L^{+}$ and the conditions $\left|L^{+}\right|=1$ and $u_{i} \geqslant \mu$ for $i \in L^{+}$are clearly necessary and sufficient.

\subsection{Lifting flow pack inequalities}

In order to both strengthen and generalize flow pack inequality (2) for $S$, for a flow pack $\left(C^{+}, C^{-}\right)$, we let $L^{-} \subseteq N^{-} \backslash C^{-}$and $K=N^{-} \backslash\left(C^{-} \cup L^{-}\right)$, and consider the projection $S^{0}=\left\{(x, y) \in S:\left(x_{j}, y_{j}\right)=\left(1, u_{j}\right)\right.$ for all $j \in \bar{C}^{+},\left(x_{j}, y_{j}\right)=(0,0)$ for all $\left.j \in L^{-}\right\} . S^{0}$ is full-dimensional if $\mu+\sum_{i \in K} u_{i} \geqslant u_{k}$ for all $k \in N^{-} \backslash L^{-}$. The inequality

$$
\sum_{i \in L^{+}}\left(y_{i}-\min \left\{u_{i}, \mu\right\} x_{i}\right)+\sum_{i \in C^{-}}\left(u_{i}-\mu\right)^{+}\left(1-x_{i}\right)-\sum_{i \in K} y_{i} \leqslant 0
$$

is facet-defining for $\operatorname{conv}\left(S^{0}\right)$ under the conditions of Proposition 1. To lift (3) for $S$, we need to compute

$$
\begin{aligned}
f(z)=-\max & \sum_{i \in L^{+}}\left(y_{i}-\min \left\{u_{i}, \mu\right\} x_{i}\right)+\sum_{i \in C^{-}}\left(u_{i}-\mu\right)^{+}\left(1-x_{i}\right)-\sum_{i \in K} y_{i} \\
\text { s.t. } & \sum_{i \in N^{+} \backslash C^{+}} y_{i}-\sum_{i \in N^{-} \backslash L^{-}} y_{i} \leqslant b^{0}-z \\
& 0 \leqslant y_{i} \leqslant u_{i} x_{i}, \quad x_{i} \in\{0,1\}, \quad y_{i} \in \mathbb{R}, \quad i \in\left(N^{+} \backslash C^{+}\right) \cup\left(N^{-} \backslash L^{-}\right),
\end{aligned}
$$

for $z \in \mathbb{R}_{-}$, where $b^{0}=b-\sum_{i \in C^{+}} u_{i}$.

We show that optimization problem (4) can be solved easily. Let $(\bar{x}, \bar{y})$ be an optimal solution to (4), let $Y=\left\{i \in L^{+}: \bar{x}_{i}=1\right\}$ and $Z=\left\{i \in C^{-}: \bar{x}_{i}=0\right\}$. We may assume that $Y \subseteq\left\{i \in L^{+}: u_{i}>\mu\right\}, Z \subseteq\left\{i \in C^{-}: u_{i}>\mu\right\}$, and $\bar{y}_{i}=u_{i}$ for all $i \in C^{-} \backslash Z$, otherwise, we can find a solution with the same or better objective value satisfying these assumptions. For the moment, assume that $\bar{y}_{i}=0$ for all $i \in K$ in an optimal solution. Then, $\sum_{i \in L^{+}} \bar{y}_{i}$ equals either $b^{0}-z+\sum_{i \in C^{-} \backslash Z} u_{i}$ or $\sum_{i \in Y} u_{i}$, depending on whether the balance constraint is tight 
or not. If $b^{0}-z+\sum_{i \in C^{-} \backslash Z} u_{i} \leqslant \sum_{i \in Y} u_{i}$ (equivalently, $-\sum_{i \in Y \cup Z} u_{i}+\mu \leqslant z$ ), then

$$
f(z)^{1}=-\left(b-\sum_{i \in C^{+}} u_{i}+\sum_{i \in C^{-} \backslash Z} u_{i}+z-|Y| \mu+\sum_{i \in Z}\left(u_{i}-\mu\right)\right)=(|Y \cup Z|-1) \mu+z .
$$

If $b^{0}-z+\sum_{i \in C^{-} \backslash Z} u_{i}>\sum_{i \in Y} u_{i}$ (equivalently, $-\sum_{i \in Y \cup Z} u_{i}+\mu>z$ ), then

$$
f(z)^{2}=-\left(\sum_{i \in Y} u_{i}-|Y| \mu+\sum_{i \in Z}\left(u_{i}-\mu\right)\right)=-\sum_{i \in Y \cup Z}\left(u_{i}-\mu\right) .
$$

Now, we argue that there exists an optimal solution such that $u_{\underline{\underline{l}}}=\min \left\{u_{i}: i \in Y \cup Z\right\} \geqslant u_{\bar{\imath}}=\max \left\{u_{i}: i \in\left(L^{+} \cup\right.\right.$ $\left.\left.C^{-}\right) \backslash(Y \cup Z)\right\}$. For contradiction, suppose $u_{\underline{l}}<u_{\bar{l}}$. If the balance constraint is tight (Case 1 ), by exchanging $\underline{l}$ and $\bar{l}$ we obtain a solution with the same objective value, since $|Y \cup Z|$ remains unchanged. Otherwise (Case 2 ), we again exchange $\underline{l}$ and $\bar{l}$. If the new solution is still in Case $2, f(z)^{2}$ decreases by $u_{\bar{l}}-u_{\underline{l}}$. If the new solution is in Case 1, the objective value decreases again since $f(z)^{2}-f(z)^{1}=-\sum_{i \in Y \cup Z}\left(u_{i}-\mu\right)-(\mid Y \cup$ $Z \mid-1) \mu-z=-\sum_{i \in Y \cup Z} u_{i}+\mu-z>0$.

Therefore, we may assume that $Y \cup Z$ consists of the first $|Y \cup Z|$ elements of $\left\{i_{1}, i_{2}, \ldots, i_{r}\right\} \equiv\left\{i \in C^{-} \cup\right.$ $\left.L^{+}: u_{i}>\mu\right\}$ indexed in nonincreasing order of $u_{i}$. Then the lifting function $f(z)$ can be expressed in a closed form as

$$
f(z)= \begin{cases}k \mu+z, \quad-w_{k+1}+\mu<z \leqslant-w_{k}, & k=0,1, \ldots, r-1, \\ k \mu-w_{k}, \quad-w_{k}<z \leqslant-w_{k}+\mu, & k=1,2, \ldots, r \\ r \mu-w_{r}, \quad z \leqslant-w_{r}, & \end{cases}
$$

where $w_{0}=0, w_{k}=\sum_{t=1}^{k} u_{i_{t}}$ for $k=1,2, \ldots, r$.

At this time, we remove the assumption that $\bar{y}_{i}=0$ for all $i \in K$. Suppose $\bar{y}_{i}>0$ for some $i \in K$. Then, the balance constraint must be tight, otherwise, a better solution is available by decreasing $\bar{y}_{i}$. We can assume that $\bar{y}_{i}=0$ for all $i \in L^{+}$, since otherwise, we can obtain a solution with the same objective value or better by decreasing both $\sum_{i \in K} \bar{y}_{i}$ and $\sum_{i \in L^{+}} \bar{y}_{i}$ in the same amount and setting $x_{i}=0, i \in L^{+}$if applicable. Therefore, $\sum_{i \in K} \bar{y}_{i}=-b^{0}+z-\sum_{i \in C^{-} \backslash Z} u_{i}>0$, which is true only if $b^{0}=b-\sum_{i \in C^{+}} u_{i}<0$. Then for such a solution, $f(z)$ equals

$$
f(z)^{3}=-\left(\sum_{i \in Z}\left(u_{i}-\mu\right)+b-\sum_{i \in C^{+}} u_{i}-z+\sum_{i \in C^{-} \backslash Z} u_{i}\right)=(|Z|-1) \mu+z .
$$

Let $\bar{Z}$ give the minimum value $\bar{f}(z)^{3} . \bar{Z}$ consists of the elements of $C^{-}$ordered in nondecreasing $u_{i}$ such that $b^{0}-z+\sum_{i \in C^{-} \backslash \bar{Z}} u_{i}<0$. Since $b^{0}-z+\sum_{i \in C^{-} \backslash \bar{Z}} u_{i}<0 \leqslant \sum_{i \in C^{+}} u_{i}$, it follows that $z>-\sum_{i \in \bar{Z}} u_{i}+\mu$, implying $f(z) \leqslant \bar{f}(z)^{3}$. It remains to check the feasibility of the solution described by $f(z)$. If $\max _{i \in L^{+}} u_{i} \leqslant \min _{i \in \bar{Z}} u_{i}$, then $f(z)$ is determined by only $\bar{Z}$. In this case, if $-\sum_{i \in \bar{Z}} u_{i}+\min _{i \in \bar{Z}} u_{i} \leqslant z$, then by definition of $\bar{Z}$, $b^{0}-z+\sum_{i \in C^{-} \backslash \bar{Z}} u_{i}+\min _{i \in \bar{Z}} u_{i} \geqslant 0$ and hence the solution is feasible (when $y_{i}=0$ for all $i \in K$ ). Else if $-\sum_{i \in \bar{Z}} u_{i}+\mu<z<-\sum_{i \in \bar{Z}} u_{i}+\min _{i \in \bar{Z}} u_{i}$, then $f(z)=(|\bar{Z}|-1) \mu+z=\bar{f}(z)^{3}$. If $\max _{i \in L^{+}} u_{i}>\min _{i \in \bar{Z}} u_{i}$, then $f(z)$ is determined by a subset of $L^{+}$and a strict subset $Z$ of $\bar{Z}$ and since $b^{0}-z+\sum_{i \in C^{-} \backslash Z} u_{i} \geqslant 0$ for $Z \subset \bar{Z}$, the solution is feasible. This completes the characterization of the lifting function $f(z)$.

It can be shown that $f$ is superadditive on $\mathbb{R}_{-}$, which implies that the lifting is sequence independent $[6,14]$, that is the lifting function $f(z)$ remains unchanged as the projected variable pairs $\left(x_{j}, y_{j}\right), j \in C^{+} \cup L^{-}$ are introduced to inequality (3) sequentially. 
In order to write the lifted pack inequalities explicitly and show their relation to the lifted flow cover inequalities, we define the following function. For $u \in \mathbb{R}_{+}$, let

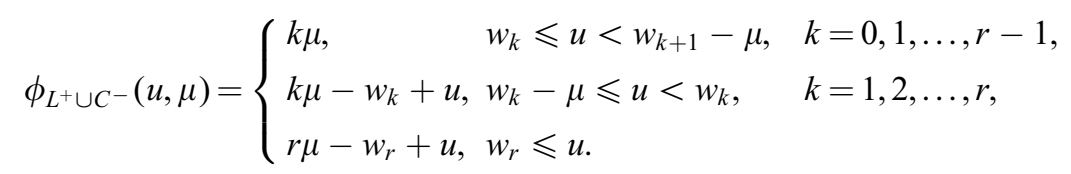

From Theorem 10 of Gu et al. [5], it can be seen that $\phi_{C^{+} \cup L^{-}}$is an approximate superadditive lifting function for flow cover inequalities. Then, after adding a slack variable $s$ to the balance constraint of $S$ and relaxing it to $\sum_{i \in N^{-}} y_{i}-\sum_{i \in N^{+}} y_{i}-s \leqslant-b$, by writing a lifted flow cover inequality for this relaxation, one obtains the following lifted flow pack inequality.

For $i \in L^{-}$, let $l=\operatorname{argmax}_{0 \leqslant h \leqslant r}\left\{u_{i} \geqslant w_{h}-\mu\right\}$. If $l=0$, then let $G_{i}=\{(-1,0)\}$. If $l>0$, then $G_{i}=$ $\{(-1,0)\} \cup G_{i}^{1} \cup G_{i}^{2}$, where

$$
G_{i}^{1}=\left\{\left(\frac{\mu}{u_{k}}-1, \mu\left(k-1-\frac{w_{k}-\mu}{u_{k}}\right)\right): k=2,3, \ldots, l\right\}
$$

and

$$
G_{i}^{2}= \begin{cases}\emptyset & \text { if } u_{i}=w_{l}-\mu, \\ \left(0, l \mu-w_{l}\right) & \text { if } w_{l}-\mu<u_{i} \leqslant w_{l} \text { or } u_{i}>w_{r}, \\ \left(\frac{\mu}{u_{i}-w_{l}+\mu}-1, \mu\left(l-\frac{u_{i}}{u_{i}-w_{l}+\mu}\right)\right) & \text { if } u_{i} \leqslant w_{r} \text { and } w_{l}<u_{i}<w_{l+1}-\mu .\end{cases}
$$

Theorem 2. Let $\left(C^{+}, C^{-}\right)$be a flow pack. If $S^{0}$ is full-dimensional, $\left(\alpha_{i}, \beta_{i}\right) \in G_{i}$ for $i \in L^{-}$, and the conditions of Proposition 1 are satisfied, then the lifted flow pack inequality

$$
\begin{aligned}
& \left.\sum_{i \in C^{+}}\left(y_{i}+\phi_{L^{+} \cup C^{-}}\left(u_{i}, \mu\right)\right)\left(1-x_{i}\right)\right)+\sum_{i \in L^{+}}\left(y_{i}-\mu x_{i}\right) \\
& \quad+\sum_{i \in C^{-}}\left(u_{i}-\min \left\{u_{i}, \mu\right\}\right)^{+}\left(1-x_{i}\right)+\sum_{i \in L^{-}}\left(\alpha_{i} y_{i}+\beta_{i} x_{i}\right)-\sum_{i \in K} y_{i} \leqslant \sum_{i \in C^{+}} u_{i},
\end{aligned}
$$

is facet-defining for $\operatorname{conv}(S)$.

Inequality (6) is facet-defining for $\operatorname{conv}(S)$, because the exact lifting function (5) for the flow pack inequalities can be written as $f(-u)=\phi_{L^{+} \cup C^{-}}(u)-u$.

\subsection{Comparison of valid inequalities}

In contrast to the lifting function of the flow pack inequality (2), the lifting function of the flow cover inequality (1) is not superadditive and its lifting is not sequence independent [5]. An intuitive explanation for the absence of superadditivity in the cover lifting function is that the nonincreasing order of $u_{i}$ may need to be violated to achieve feasibility when lifting a flow cover inequality; the lifting problem of flow cover inequalities is a restriction, whereas the lifting problem of flow pack inequalities is a relaxation.

In order to highlight the relation among valid inequalities for $S$, we write a form of the lifted flow cover inequalities, which uses $\phi_{C^{+} \cup L^{-}}$as a valid superadditive lifting function, even though this function is slightly weaker than the one given in Theorem 11 of Gu et al. [5] for flow covers. Let $\left(C^{+}, C^{-}\right)$be a flow cover. If 
$\left(\alpha_{i}, \beta_{i}\right) \in G_{i}$ for $i \in L^{+}$, then the following lifted flow cover inequality is valid for $S$ :

$$
\begin{aligned}
& \sum_{i \in C^{+}}\left(y_{i}+\left(u_{i}-\lambda\right)^{+}\left(1-x_{i}\right)\right)+\sum_{i \in L^{+}}\left(\left(\alpha_{i}+1\right) y_{i}+\beta_{i} x_{i}\right) \\
& \quad+\sum_{i \in C^{-}} \phi_{C^{+} \cup L^{-}}\left(u_{i}, \lambda\right)\left(1-x_{i}\right)-\sum_{i \in L^{-}} \min \left\{u_{i}, \lambda\right\} x_{i}+\sum_{i \in K} y_{i} \leqslant b+\sum_{i \in C^{-}} u_{i} .
\end{aligned}
$$

Now we compare the lifted flow pack and the lifted flow cover inequalities with the $k$-cover and $k$-reversecover inequalities [7] derived from mixed 0-1 knapsack relaxations of $S$. Observing that $\phi_{L^{+} \cup C^{-}}(u, \mu)+$ $\psi_{L^{+} \cup C^{-}}(u)=u(\psi$ is a notation used in [7]), after algebraic manipulations, the $k$-cover inequality can be restated as

$$
\begin{aligned}
& \sum_{i \in C^{+}}\left(y_{i}-\left(u_{i}-\lambda\right)^{+} x_{i}\right)+\sum_{i \in L^{+}}\left(y_{i}-\left(u_{i}-\phi_{C^{+} \cup L^{-}}\left(u_{i}, \lambda\right)\right) x_{i}\right) \\
& \quad+\sum_{i \in C^{-}} \phi_{C^{+} \cup L^{-}}\left(u_{i}, \lambda\right)\left(1-x_{i}\right)-\sum_{i \in L^{-}} \min \left\{u_{i}, \lambda\right\} x_{i}-\sum_{i \in K} y_{i} \leqslant b+\sum_{i \in C^{-}} u_{i}
\end{aligned}
$$

and the k-reverse-cover inequality can be restated as

$$
\begin{aligned}
& \sum_{i \in C^{+}}\left(y_{i}+\phi_{L^{+} \cup C^{-}}\left(u_{i}, \mu\right)\left(1-x_{i}\right)\right)+\sum_{i \in L^{+}}\left(y_{i}-\min \left\{u_{i}, \mu\right\} x_{i}\right) \\
& \quad+\sum_{i \in C^{-}}\left(u_{i}-\mu\right)^{+}\left(1-x_{i}\right)-\sum_{i \in L^{-}}\left(u_{i}-\phi_{L^{+} \cup C^{-}}\left(u_{i}, \mu\right)\right) x_{i}-\sum_{i \in K} y_{i} \leqslant \sum_{i \in C^{+}} u_{i} .
\end{aligned}
$$

Lifted flow pack inequality (6) with $\left(\alpha_{i}, \beta_{i}\right) \in G_{i}^{2}$ for all $i \in L^{+}$is at least as strong as $k$-reverse-cover inequality (9). To see this, observe that for $i \in L^{+}$, if $w_{l}-\mu<u_{i} \leqslant w_{l}$ or $u_{i}>w_{r}$, then $\beta_{i}=l \mu-w_{l}=\phi_{C^{+} \cup L^{-}}\left(u_{i}, \lambda\right)$, and thus the inequalities are the same. Else if $u_{i} \leqslant w_{r}$ and $w_{l}<u_{i}<w_{l+1}-\mu$, then for $(\bar{x}, \bar{y}) \in S$, let $\zeta_{1}=\alpha_{i} \bar{x}_{i}+\beta_{i} \bar{y}_{i}=\mu\left(l-u_{i} / u_{i}-w_{l}+\mu\right) \bar{x}_{i}+\left(\mu / u_{i}-w_{l}+\mu\right) \bar{y}_{i}$ and $\zeta_{2}=-\left(u_{i}-\phi_{L^{+} \cup C^{-}}\left(u_{i}, \mu\right)\right) \bar{x}_{i}=\left(l \mu-u_{i}\right) \bar{x}_{i}$. Then $\zeta_{1}-\zeta_{2}=\left(1-\mu / u_{i}-w_{l}+\mu\right)\left(u_{i} \bar{x}_{i}-\bar{y}_{i}\right) \geqslant 0$. Similarly, lifted flow cover inequality (7) is at least as strong as $k$-cover inequality (8) for $S$. Yet, it is still very interesting that one gets strong valid inequalities such as (8) and (9) for $S$ from its much simpler mixed 0-1 knapsack relaxation.

Example. Suppose $S$ is given by

$$
\begin{aligned}
& y_{1}+y_{2}+y_{3}+y_{4}-y_{5}-y_{6}-y_{7} \leqslant 3 \\
& y_{1} \leqslant 6 x_{1}, \quad y_{2} \leqslant 4 x_{2}, \quad y_{3} \leqslant 6 x_{3}, \quad y_{4} \leqslant 8 x_{4}, \quad y_{5} \leqslant 5 x_{5}, \quad y_{6} \leqslant 2 x_{6}, \quad y_{7} \leqslant 12 x_{7} .
\end{aligned}
$$

Let $C^{+}=\{1\}, C^{-}=\{5\}, L^{+}=\{2,3\}, L^{-}=\{7\}$, and $K=\{6\}$. Then $\mu=2$ and $y_{2}-2 x_{2}+y_{3}-2 x_{3}+3(1-$ $\left.x_{5}\right)-y_{6} \leqslant 0$ is valid for $S^{0}=\left\{(x, y) \in S:\left(x_{1}, y_{1}\right)=(1,6),\left(x_{7}, y_{7}\right)=(0,0)\right\}$. Note that $w_{0}=0, w_{1}=6, w_{2}=11$, $w_{3}=15$. Lifting it with $x_{1}, y_{1}, x_{7}$, and $y_{7}$, we obtain the following lifted flow pack inequalities:

$$
\begin{aligned}
& y_{1}+2\left(1-x_{1}\right)+y_{2}-2 x_{2}+y_{3}-2 x_{3}-3\left(1-x_{5}\right)-y_{6}-y_{7}-0 x_{7} \leqslant 6, \\
& y_{1}+2\left(1-x_{1}\right)+y_{2}-2 x_{2}+y_{3}-2 x_{3}-3\left(1-x_{5}\right)-y_{6}-\frac{3}{5} y_{7}-\frac{8}{5} x_{7} \leqslant 6, \\
& y_{1}+2\left(1-x_{1}\right)+y_{2}-2 x_{2}+y_{3}-2 x_{3}-3\left(1-x_{5}\right)-y_{6}-\frac{1}{3} y_{7}-4 x_{7} \leqslant 6 .
\end{aligned}
$$

Alternatively, let $C^{+}=\{1,2\}, C^{-}=\{5\}, L^{+}=\{4\}, L^{-}=\emptyset$, and $K=\{6,7\}$. Then $\lambda=2$ and $y_{1}+4(1-$ $\left.x_{4}\right)+y_{2}+2\left(1-x_{2}\right)-y_{6}-y_{7} \leqslant 8$ is valid for $S^{0}=\left\{(x, y) \in S:\left(x_{4}, y_{4}\right)=(0,0),\left(x_{5}, y_{5}\right)=(1,5)\right\}$. Here, $w_{0}=0, w_{1}=6, w_{2}=10$. Lifting it with $x_{4}, y_{4}, x_{5}$, and $y_{5}$, we get the following lifted flow cover inequalities:

$$
\begin{aligned}
& y_{1}+4\left(1-x_{1}\right)+y_{2}+2\left(1-x_{2}\right)+0 y_{4}-0 x_{4}-\left(1-x_{5}\right)-y_{6}-y_{7} \leqslant 8, \\
& y_{1}+4\left(1-x_{1}\right)+y_{2}+2\left(1-x_{2}\right)+\frac{1}{2} y_{4}-2 x_{4}-\left(1-x_{5}\right)-y_{6}-y_{7} \leqslant 8 .
\end{aligned}
$$




\section{Computational results}

In this section, we present a summary of computational experiments on using the lifted flow pack inequalities (6) in a branch-and-cut algorithm for solving capacitated fixed-charge network flow problems. The branch-and-cut algorithm was implemented with MINTO [8] (version 3.0) using CPLEX (version 6.0) as the LP solver. All experiments were performed on a SUN Ultra 10 workstation. In addition to the lifted pack inequalities, we used the lifted flow cover inequalities that are automatically generated by MINTO. The lifted flow cover inequalities that MINTO generates are similar to inequality (7) and are described in $\mathrm{Gu}$ et al. [5].

In order to test the effectiveness of the lifted flow pack inequalities, we ran the branch-and-cut algorithm on a set of randomly generated capacitated fixed-charge network flow problems available at http://ieor. berkeley.edu/ atamturk/data. In Tables 1 and 2, we present a comparison of the performance of the branch-and-cut algorithm when run with only MINTO's lifted flow cover inequalities, by adding flow pack inequalities, and finally by adding lifted flow pack inequalities. Table 1 is for instance with 30 nodes and $50 \%$ arc density, whereas Table 2 is for instance with 60 nodes and $20 \%$ arc density. For this experiment, we also implemented a simple primal heuristic, which rounds fractional binary variables in LP solutions in the search tree to one, in order to construct feasible solutions quickly for pruning. We report the objective values of the initial LP relaxation after preprocessing (zinit), optimal integral solution (zopt), and the LP relaxation at the root node of the search tree after all cuts are added (zroot), the number of lifted flow cover cuts (lfcovs), the number of flow pack cuts (fpacks), the number of lifted flow pack cuts (lfpacks), the number of nodes evaluated (nodes), and the CPU time elapsed in seconds. All fractional entries are rounded to the nearest integer. A comparison of the entries in columns zinit and zopt shows that all of these problems have big duality gaps. LP relaxations at the root node (zroot) improve consistently with the addition of flow pack and lifted flow pack cuts. We also see significant reductions in the number of nodes explored and in the CPU time elapsed. Note that more lifted flow pack cuts are generated than flow pack cuts and that lifting flow pack cuts do improve the LP relaxations. The effect of lifting flow pack inequalities is more significant for the larger instances in Table 2 . These computational results indicate that the flow pack inequalities and the lifted flow pack inequalities improve the performance of the branch-and-cut algorithm significantly for capacitated fixed-charge network flow problems.

Table 1

Computations with capacitated fixed-charge network flow problems: 30 nodes

\begin{tabular}{|c|c|c|c|c|c|c|c|c|c|c|c|c|c|c|c|c|}
\hline \multirow[b]{2}{*}{ Problem } & \multirow[b]{2}{*}{ zinit } & \multirow[b]{2}{*}{ zopt } & \multicolumn{4}{|c|}{ Without fpacks } & \multicolumn{5}{|c|}{ With fpacks } & \multicolumn{5}{|c|}{ With lfpacks } \\
\hline & & & zroot & lfcovs & nodes & time & zroot & lfcovs & fpacks & nodes & time & zroot & lfcovs & 1fpacks & nodes & time \\
\hline fc. 30.1 & 120 & 307 & 259 & 458 & 9783 & 210 & 261 & 286 & 45 & 5434 & 122 & 261 & 309 & 186 & 6320 & 145 \\
\hline fc. 30.2 & 152 & 325 & 273 & 341 & 4689 & 95 & 276 & 334 & 75 & 1807 & 38 & 280 & 230 & 171 & 1412 & 43 \\
\hline fc. 30.3 & 98 & 294 & 235 & 252 & 3872 & 47 & 251 & 412 & 125 & 2193 & 68 & 254 & 245 & 159 & 1820 & 53 \\
\hline fc. 30.4 & 100 & 763 & 712 & 261 & 3238 & 40 & 734 & 305 & 65 & 2213 & 35 & 738 & 205 & 104 & 1847 & 28 \\
\hline fc. 30.5 & 158 & 301 & 274 & 146 & 215 & 4 & 277 & 172 & 36 & 145 & 4 & 277 & 128 & 60 & 149 & 4 \\
\hline fc. 30.6 & 126 & 272 & 238 & 296 & 8759 & 105 & 241 & 276 & 64 & 3019 & 72 & 245 & 243 & 187 & 4357 & 93 \\
\hline fc. 30.7 & 97 & 231 & 204 & 187 & 1471 & 14 & 207 & 167 & 30 & 458 & 8 & 212 & 176 & 98 & 554 & 10 \\
\hline fc. 30.8 & 176 & 347 & 300 & 431 & 13623 & 270 & 305 & 381 & 72 & 4257 & 113 & 305 & 290 & 182 & 6063 & 147 \\
\hline fc. 30.9 & 85 & 741 & 708 & 334 & 3172 & 56 & 709 & 257 & 43 & 1818 & 37 & 710 & 313 & 145 & 1793 & 40 \\
\hline fc. 30.10 & 93 & 204 & 171 & 252 & 2991 & 32 & 173 & 249 & 66 & 1217 & 17 & 179 & 166 & 118 & 507 & 11 \\
\hline
\end{tabular}


Table 2

Computations with capacitated fixed-charge network flow problems: 60 nodes

\begin{tabular}{|c|c|c|c|c|c|c|c|c|c|c|c|c|c|c|c|c|}
\hline \multirow[b]{2}{*}{ Problem } & \multirow[b]{2}{*}{ zinit } & \multirow[b]{2}{*}{ zopt } & \multicolumn{4}{|c|}{ Without fpacks } & \multicolumn{5}{|c|}{ With fpacks } & \multicolumn{5}{|c|}{ With lfpacks } \\
\hline & & & zroot & lfcovs & nodes & time & zroot & lfcovs & lfpacks & nodes & time & zroot & lfcovs & lfpacks & nodes & time \\
\hline fc. 60.1 & 171 & 487 & 394 & 612 & 79448 & 2062 & 413 & 751 & 225 & 23631 & 1122 & 415 & 468 & 350 & 21444 & 1054 \\
\hline fc. 60.2 & 230 & 584 & 445 & 830 & 82050 & 1018 & 459 & 975 & 347 & 33580 & 1812 & 490 & 471 & 450 & 15070 & 634 \\
\hline fc. 60.3 & 161 & 493 & 414 & 494 & 11124 & 300 & 420 & 698 & 194 & 15091 & 497 & 420 & 358 & 279 & 4929 & 150 \\
\hline fc. 60.4 & 120 & 442 & 337 & 780 & 141882 & 4175 & 354 & 999 & 322 & 30956 & 1652 & 354 & 532 & 423 & 19386 & 960 \\
\hline fc. 60.5 & 160 & 414 & 332 & 451 & 20436 & 309 & 336 & 587 & 155 & 9775 & 238 & 349 & 259 & 245 & 1734 & 39 \\
\hline fc. 60.6 & 199 & 480 & 388 & 681 & 127285 & 3656 & 399 & 738 & 237 & 28929 & 1063 & 421 & 491 & 373 & 20553 & 939 \\
\hline fc. 60.7 & 177 & 492 & 422 & 463 & 7450 & 184 & 426 & 497 & 101 & 5350 & 373 & 443 & 375 & 305 & 4090 & 176 \\
\hline fc. 60.8 & 202 & 500 & 440 & 486 & 14360 & 353 & 453 & 671 & 159 & 7897 & 425 & 455 & 438 & 330 & 5239 & 289 \\
\hline fc. 60.9 & 164 & 397 & 358 & 342 & 3620 & 54 & 365 & 312 & 47 & 1557 & 25 & 365 & 227 & 142 & 915 & 16 \\
\hline fc. 60.10 & 180 & 913 & 823 & 820 & 44523 & 1982 & 827 & 759 & 196 & 8675 & 508 & 834 & 450 & 255 & 11912 & 534 \\
\hline
\end{tabular}

\section{Acknowledgements}

I am grateful to Z. Gu, G.L. Nemhauser and M.W.P. Savelsbergh for their valuable comments and to L.A. Wolsey for pointing out that flow cover inequalities can be used to show the validity of flow pack inequalities, which shortened the presentation significantly.

\section{References}

[1] K. Aardal, Y. Pochet, L.A. Wolsey, Capacitated facility location: valid inequalities and facets, Math. Oper. Res. 20 (1995) $562-582$.

[2] A. Atamtürk, Flow pack facets of the single node fixed-charge flow polytope, Research Report BCOL99.01, University of California at Berkeley, Berkeley, USA, 1999, available at http://ieor.berkeley.edu/ atamturk.

[3] I. Barany, T.J. Van Roy, L.A. Wolsey, Uncapacitated lot sizing: the convex hull of solutions, Math. Programming Stud. 22 (1984) $32-43$.

[4] C. Cordier, H. Marchand, R. Laundy, L.A. Wolsey, bc-opt: a branch-and-cut code for mixed integer programs, Math. Programming 86 (1999) 335-354.

[5] Z. Gu, G.L. Nemhauser, M.W.P. Savelsbergh, Lifted flow cover inequalities for mixed 0-1 integer programs, Math. Programming 85 (1999) 439-467.

[6] Z. Gu, G.L. Nemhauser, M.W.P. Savelsbergh, Sequence independent lifting in mixed integer programming, J. Combin. Optim. 4 (2000) 109-129.

[7] H. Marchand, L.A. Wolsey, The 0-1 knapsack problem with a single continuous variable, Math. Programming 85 (1999) 15-33.

[8] G.L. Nemhauser, M.W.P. Savelsbergh, G.S. Sigismondi, MINTO, a mixed integer optimizer, Oper. Res. Lett. 15 (1994) $47-58$.

[9] M.W. Padberg, T.J. Van Roy, L.A. Wolsey, Valid linear inequalities for fixed charge problems, Oper. Res. 32 (1984) $842-861$.

[10] Y. Pochet, Valid inequalities and separation for capacitated economic lot sizing, Oper. Res. Lett. 7 (1988) $109-115$.

[11] J.I.A. Stallaert, The complementary class of generalized flow cover inequalities, Discrete Appl. Math. 77 (1997) 73-80.

[12] T.J. Van Roy, L.A. Wolsey, Valid inequalities for mixed 0-1 programs, Discrete Appl. Math. 14 (1986) 199-213.

[13] T.J. Van Roy, L.A. Wolsey, Solving mixed integer programming problems using automatic reformulation, Oper. Res. 35 (1987) $45-57$.

[14] L.A. Wolsey, Valid inequalities and superadditivity for 0/1 integer programs, Math. Oper. Res. 2 (1977) 66-77. 